Öko-Effizienz als Element des sozio-ökonomisch vernünftigen Umweltmanagements

\section{Ein Kriterium unter vielen}

\section{Die Grundlage ökologisch vernünftigen Handelns ist eine bestimmte}

Denkhaltung. Aber was ist ökologisch vernünftiges Handeln im Kontext der Unternehmungsführung? Der vorliegende Beitrag wiederbelebt das heuristische Konzept des sozio-ökonomisch vernünftigen Managements von Umweltproblemen. In diesem Rahmen wurde der Ansatz der Öko-Effizienz als eines von fünf Erfolgskriterien des normativen Umwelimanagements erstmals diskutiert (1). Dabei ist vor einer isolierten Betrachfung und Überbetonung des ÖkoEffizienz-Kriteriums zu warnen.

$\mathrm{D}$ Von Stefan Schaltegger ie Lenkung von Unternehmungen ist zumindest zum Teil eine externe. Dabei lassen sich fünf externe Lenkungssysteme von Organisationen mit jeweils verschiedenen Erfolgskriterien unterscheiden (2): Moral, Recht, Technologie, Markt und Politik (vgl. Abb. 1).

\begin{tabular}{|c|c|c|c|c|c|}
\hline \multicolumn{6}{|c|}{ Abbildung 1: Das Konzept der sozio-ökonomischen Rationalitäi } \\
\hline Lenkungssystem & Moral & Recht & Technologie & Markt & Politik \\
\hline Anspruch & Ziel & $\begin{array}{l}\text { Rahmen- } \\
\text { bedingungen }\end{array}$ & $\begin{array}{l}\text { Zielerrei: } \\
\text { chungsgrad }\end{array}$ & Output zu Input & Durchselzung \\
\hline betreffend & $\begin{array}{l}\text { Welches Ziel } \\
\text { soll verfolgt } \\
\text { werden? } \\
\text { Werthaltungen } \\
\text { \& Normen }\end{array}$ & $\begin{array}{c}\text { Welche Rahmen- } \\
\text { bedingungen sollen } \\
\text { den Akteuren } \\
\text { gesetzt werden? }\end{array}$ & $\begin{array}{l}\text { Wie gut wird } \\
\text { dos Ziel } \\
\text { erreicht? }\end{array}$ & $\begin{array}{l}\text { Mit welchem } \\
\text { Aufwand-Ertrogs- } \\
\text { Verhälthis wird das } \\
\text { Ziel erreicht? }\end{array}$ & $\begin{array}{l}\text { Wie wird die } \\
\text { Zielerreichung } \\
\text { verfolgt? }\end{array}$ \\
\hline Effolgskriterium & Legitimitöit & Legalitöt & Effektivität & Effizienz & $\begin{array}{l}\text { Handlungsspiel } \\
\text { raum \& Machi }\end{array}$ \\
\hline Rationalität & soziokulturell & juristisch & technologisch & wirtschaftllich & politisch \\
\hline
\end{tabular}

Quelle: eigene Darstellung in Anlehnung an Schaltegger/ Sturm 1990 (vgl. Anmerkung 1)

Die relative Bedeutung dieser Lenkungssysteme ist je nach Land, Kulturkreis und Zeitpunkt unterschiedlich. Die Sicherung der gesellschaftlichen Legitimation, als Voraussetzung einer gesunden Entwicklung einer Unternehmung, erfordert ein erfolgreiches Manövrieren in allen Lenkungssystemen bzw. ein sozioökonomisch rationales Verhalten des Managements (3), das den Kriterien der soziokulturellen, juristischen, technischen, wirtschaftlichen und politischen Rationalität genügt. Diese fünf Dimensionen des heuristischen Konzepts werden im folgenden aus der Sicht der Unternehmensleitung dargelegt.
Ziele und gehen der zentralen Fragestellung nach, welche Ziele von einer Unternehmung verfolgt werden sollen.

Je nach Land bestehen unterschiedliche Werthaltungen zum Umweltschutz, zur relativen Bedeutung einzelner Probleme und darüber, was sinnvolle Schutzmaßnahmen darstellen. So ist z.B. Littering in vielen Ländern ein verbreitetes, sozial akzeptiertes Phänomen (z.B. Türkei) während es in anderen Ländern (z.B. Singapur) stark geächtet wird. Das Konzept der nachhaltigen Entwicklung findet dennoch weltweit eine immer breitere Unterstïtzung in Gesellschaft und Politik.
Das Erfolgskriterium im soziokulturellen Umfeld ist die Legitimität. Ein soziokulturell rationales Umweltmanagement kennzeichnet sich dadurch, dass es den ökologischen Werthaltungen eines Kulturkreises Rechnung trägt. Dies darf nicht mit einer Anpassung an die schwächsten Umweltschutzanforderungen verwechselt werden, sondern bedeutet, dass zur Sicherung eines wirksamen Vollzugs bei der Ausgestaltung von Maßnahmen auf die spezifischen gesellschaftlichen Sitten Rücksicht genommen werden muss.

\section{Öko-Legalitäł}

In entwickelten Ländern wurden viele Bräuche und Sitten kodifiziert und ein eigenes Lenkungssystem - dasjenige des Rechts - geschaffen. Das juristische Lenkungssystem übt über Rechtswerke und -organisationen wie Gesetzesbücher und Gerichte Einfluss auf die Handlungen von Individuen aus. Zentrale Fragestellung im rechtlichen Umfeld der Unternehmung ist, welche Rahmenbedingungen dem Management gesetzt werden sollen. Die Veränderung der rechtlichen Rahmenbedingungen ist die dominierende Strategie, Unternehmungen zu mehr Umweltschutz zu bewegen.

Das Rechtssystem und die gelebte soziokulturelle Kultur können sehr unterschiedlich sein. Dabei müssen die rechtssetzenden Instanzen immer wieder feststellen, dass das Rechtssystem sich langfristig nicht wirksam über das soziokulturelle Wertesystem stülpen lässt. Ein juristisch rationales Umweltmanagement zeichnet sich daher durch Konformität mit dem Umweltrecht, also durch Öko-Legalität aus.

\section{Öko-Effektivität}

Die zentrale Fragestellung im von technologischen und wissenschaftlichen Entwicklungen geprägten Umfeld ist, wie gut das angestrebte Ziel erreicht wird. Dabei ist Effektivität die Maßgröße der Zielerreichung bzw. Wirksamkeit. Unter ökologischer Effektivität (ÖkoEffektivität) wird die Wirksamkeit von Schutzmaßnahmen und der Grad der absoluten Umweltverträglichkeit unternehmerischer Handlungen verstanden (4). Öko-Effektivität wird durch die Art der Aktivität und die in Anspruch genommene Menge beeinflusst.

Ein technologisch rationales Umweltmanagement kennzeichnet sich dadurch, dass es die Öko-Effektivität der Umweltschutzmassnahmen erhöht und die gesamte Umweltbelastung der 
Unternehmung und ihrer Leistungen reduziert. Öko-Effektivitäts-Betrachtungen können dabei für unterschiedliche Systeme (Produkte, Produktionsprozesse usw.), Zeitdimensionen und aus Sicht unterschiedlicher Stakeholder erfolgen.

\section{Öko-Effizienz}

Das Erfolgskriterium im Markt ist die Effizienz. Da Inputfaktoren knapp sind, ist Effizienz, als das Verhältnis von bewertetem Output zu bewertetem Input, das traditionell im Zentrum stehende „Muss-Kriterium“ zur Sicherung der Existenz der Unternehmung. Die betriebliche Effizienz wird i.d.R. als Rentabilität bzw. als Verhältnis von Ertrag und Leistung, bzw. Nutzen und Kosten gemessen. Da Umweltnutzung keinen oder einen zu geringen Preis hat und die physischen Verursacher von Umweltbelastungen einen Teil der Kosten ihres Verhaltens nicht tragen, ist der Wert der natürlichen Umwelt nicht in den finanzwirtschaftlichen Effizienzkennziffern berücksichtigt. Für das Umweltmanagement ist deshalb eine Ergänzung um die ökologische Effizienz und die ökonomisch-ökologische Effizienz erforderlich.

Aus einer ökologieorientierten Perspektive lässt sich analog zur Wertschöpfung die Schadschöpfung ermitteln. Schadschöpfung ist die Summe aller durch betriebliche Leistungserstellungsprozesse verursachten und bezüglich ihrer relativen Schädlichkeit gewichteten Umwelteinwirkungen. Ansätze zur Messung der Schadschöpfung basieren auf Materialflussrechmungen, Ökobilanzmethoden und dem ökologischen Rechnungswesen. Wird die generelle Definition von Effizienz als das Verhältnis von erwünschtem Output zu Input in einem rein ökologischen Sinne interpretiert, so kann von ökologischer Effizienz gesprochen werden. Die ökologische Effizienz ist die Relation zwischen erwünschter Leistung und verursachter Schadschöpfung.

Dabei sind zwei Perspektiven zu unterscheiden: - Ökologische Produkteffizienz: Verglichen wird die Schadschöpfung unterschiedlicher Produktvarianten wie z.B. verschiedener Autotypen mit vergleichbarer Leistung. Das Auto mit der geringsten gesamten Schadschöpfung hat die größte ökologische Produkteffizienz.

- Ökologische Funktionseffizienz: Verglichen wird die Schadschöpfung unterschiedlicher Produkte zur Erfüllung einer bestimmten Funktion wie z.B. die Bewältigung einer Distanz von drei Kilometern in zehn Minuten in einer Großstadt. Dabei können Straßenbahn, Auto und Fahrrad miteinander verglichen werden. Das Fahrrad verursacht die geringste Schadschöpfung und erfïllt die Funktion deshalb am ökologisch effizientesten.

Obwohl eine Verbesserung der ökologischen Produkteffizienz grundsätzlich erstrebenswert ist, stellt sie oftmals eine Suboptimierung dar. So können gewisse Produkte niemals eine so gute Funktionseffizienz erreichen wie andere (z.B. Auto versus Fahrrad im Stadtverkehr). Zur Verbesserung der Öko-Effektivität ist die Beurteilung der Funktionseffizienz deshalb notwendig. Zur Sicherstellung eines sowohl ökologisch als auch finanzwirtschaftlich effizienten Einsatzes des Umweltschutzbudgets müssen die Maßnahmen mit dem größten ökologischen Nettonutzen pro hierfür eingesetzten Geldbetrag ermittelt werden. Die Maßzahl hierfür ist die ökonomisch-ökologische Effizienz oder kurz ÖkoEffizienz. Die Beurteilung der Öko-Effizienz kann für alle, auch die nicht auf Umweltschutzbelange ausgerichteten, unternehmerischen Handlungen erfolgen. Generell steht dabei das Verhältnis der ökonomischen Leistung zur Schadschöpfung im Vordergrund:

$$
\text { Öko-Effizienz }=\frac{\text { Wertschöpfung }}{\text { Schadschöpfung }}
$$

Zusammenfassend kennzeichnet sich ein wirtschaftlich rationales Umweltmanagement durch eine Verbesserung der Öko-Effizienz der Unternehmung und unternehmerischer Handlungenl.

\section{Ökologische Interessenpolitik und Handlungsspielraum}

Stakeholder versuchen ihre ökologisch relevanten Ansprïche oft in interessenpolitischen Prozessen durchzusetzen. Eine uneingeschränkte Befriedigung der Stakeholder durch das Management ist jedoch nicht möglich, da die prinzipiell unlimitierten Ansprüche einer Knappheit von Gütern gegenuiberstehen. Das Management ist deshalb gezwungen, die Interessen gewisser Gruppen zurückzustellen. Da Stakeholder dies selten widerstandslos zulassen, kommt es zu Verteilungskämpfen. Ansprüche aus dem interessenpolitischen Umfeld der Unternehmung gehen daher der Frage nach, wie Ziele durchgesetzt werden (sollen). Die Durchsetzung von Ansprïchen kennzeichnet sich durch die Beeinflussung der Machtverteilung und des Handlungsspielraums von Stakeholdern sowie durch Aushandlungsprozesse zwischen konfligierenden Ansprüchen.

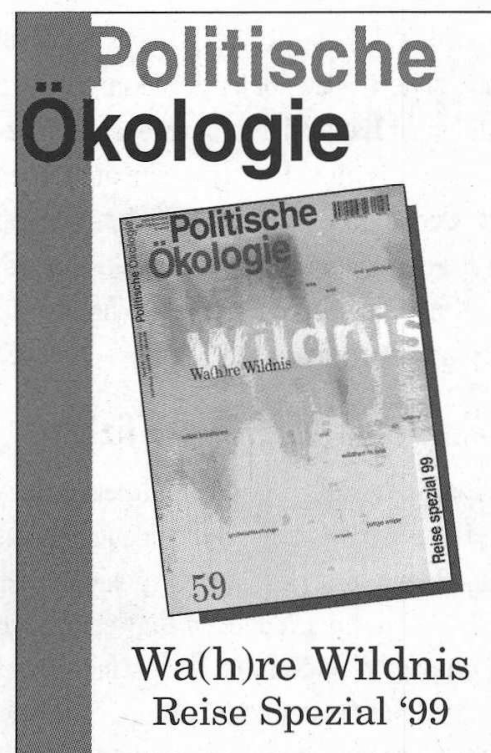

„Natur pur“ - der Trip in die Wildnis ist für viele zivilsationsmüde Städter das einzig wahre Urlaubsmotto. Nicht umsonst stehen Natur- und Abenteuerreisen hoch im Kurs.

Doch ist die touristisch genutzte Ware mit der wahren Wildnis überhaupt vereinbar?

Wieviel Wildnis wollen wir Menschen und wieviel Wildnis braucht die Natur? Die Politische Ökologie begibt sich auf Spurensuche nach der Wildnis in uns. Denn in der heutigen Zeit lauert das Wilde nicht mehr im Wald oder in der unberührten Natur sondern vielmehr in unseren Köpfen.

\section{Außerdem im Heft:}

Reise Spezial ' 99

mit

- vielen ausgewählten Reiseangeboten - beispielhaften touristischen Projekten - nützlichen Kontaktadressen - Literaturtips

Ein unverzichtbarer Ratgeber für all diejenigen, die beim nächsten Urlaub die ausgetretenen Pfade nicht $z u$ Lasten der Natur verlassen wollen.

Ausführliche Inhaltsangaben aller Ausgaben finden Sie auch auf unserer Internetseite: www.oekom.de

- ökom GmbH

- Waltherstr. 29, Rigb. - 80337 München

- Tel.: 089/544 184-0.

Fax: 089/544 184-99

- E-maill: info@oekom.de 
Macht stellt dabei die Fähigkeit eines Stakeholders dar, andere Stakeholder gegen ihren Willen $\mathrm{zu}$ bestimmten Handlungen $\mathrm{zu}$ bewegen. Interessenpolitisch rationales Umweltmanagement sichert oder vergrößert den Handlungsspielraum zur Realisierung einer nachhaltigen Gesellschaft. Hierzu kann auch die Berücksichtigung der vier anderen Kriterien beitragen.

\section{- Kein Primat der Öko-Effizienz}

Das Erfolgskriterium der Öko-Effizienz wurde einige Jahre nach seiner konzeptionellen Entwicklung von verschiedenen Exponenten als zentrales Ziel des betrieblichen Umweltmanagements propagiert und in den letzten Jahren teilweise zum alleinstehenden Imperativ des betrieblichen Umweltmanagements hochstilisiert (5). Obwohl das Konzept akademischen Ursprungs ist, wird öko-Effizienz oft als ,concept of business for business" (6) angepriesen. Mehr oder weniger willkïrliche Quantifizierungen und Spielarten der Öko-Effizienz finden sich unter den Schlagworten „Faktor vier“ (7), „Faktor zehn“ oder neuerdings „Natural Capitalism“ (8). Beim Studium der in der Literatur zuhauf aufgezählten Beispiele fallen einerseits die große Zahl sehr unterschiedlicher, teilweise konfligierender Definitionen von Öko-Effizienz auf. Auch haben viele der genannten (löblichen) Praxisbeispiele keinen klaren Bezug zur ÖkoEffizienz im Sinne einer Verhältnisbetrachtung. Ohne Zweifel: die Steigerung der Öko-Effizienz birgt ein enormes Umweltschutzpotential. Es ist für die nachhaltige Entwicklung notwendig aber nicht hinreichend. Von einem sozio-ökonomisch rationalen Umweltmanagement kann nur gesprochen werden, wenn das Management bezüglich ökologischer Anforderungen legitim, legal, effektiv und effizient handelt und die Handlungsautonomie sichert. Die relative Gewichtung der fünf Erfolgskriterien kann nicht generell bestimmt werden, sondern muss anhand der spezifischen Situation einer Unternehmung und insbesondere der Konstellationen des Stakeholdernetzwerkes beurteilt werden.

Besteht zwischen den bedeutendsten Stakeholdern ein Konsens, dass die Unternehmung vermehrt ökologischen Aspekten gerecht werden soll, so muss das Management die Erfiillung aller Erfolgskriterien des sozio-ökonomisch rationalen Umweltmanagements kontinuierlich uiberprïfen. Wichtig ist dabei, dass die Untererfiillung eines Kriteriums nicht durch die Übererfïllung eines anderen kompensiert werden kann. Wenn beispielsweise das Management die Gesetze oder ökologieorientierte Werthaltungen zuwenig berïcksichtigt und gewisse Stakeholder auf die Unternehmung deshalb Druck ausüben, so kann dem nicht wirksam durch eine Steigerung der Öko-Effizienz begegnet werden.

In der Auseinandersetzung zwischen Greenpeace und Brent Spar hat ein Vertreter von Shell in einem Interview gesagt, er begreife nicht, wie die Probleme hätten entstehen können; Shell habe die besten Techniker, Juristen und Manager. Dies verdeutlicht, dass Shell übersehen hatte, dass die Anhänger von Greenpeace die Versenkung von Ölplattformen nicht primär als ineffizient, illegal oder ineffektiv, sondern als illegitim erachteten. Das Meer ist keine Abfallkippe!

Trotz des unbestrittenen Potentials einer Faktor 4, 10 oder X Revolution ist zu beachten, dass ein monofokussiertes Öko-Effizienzmanagement immer die Gefahr laufen wird, in den anderen Erfolgsdimensionen zu versagen.

\section{Anmerkungen}

(1) Vgl. Schaltegger, S. A. Sturm: Ökologische Rationalität, in: Die Unternehmung, Nr. 4/1990, S. 273-290 sowie Schaltegger, S. A. Sturm: Ökologieorientierte Entscheidungen in Unternehmen, Bern: Haupt 1992

(2) Vgl. Dyllick, I.: Management der Umweltbeziehungen. Öffentliche Auseinandersetzung als Herausforderung, Wiesbaden: Gabler 1989.

(3) Vgl. Hill, W.: Betriebswirtschaftslehre als Managementlehre, in: Wunderer, Rolf (Hrsg.): Betriebswirtschaftslehre als Management- und Führungslehre, Stuttgart: Poeschel 1985, S. 111-146.

(4) Schaltegger/Sturm a.a.0., S. 278 und Stahlmann, V.: Öko-Effizienz und Öko-Effektivität, in: Umweltwirtschaftsforum 4/1996.

(5) Vgl. z.B. Schmidheiny, S.: Kurswechsel, München: Artemis \& Winkler 1992 und OECD : Eco-Efficiency, Paris: OECD 1998

(6) De Simone, L./ F. Popoff: Eco-Efficiency, Cambridge: MIT-Press 1997

(7) Von Weizsäcker, E.U./ A. Lovins/ H. Lovins: Faktor Vier: doppelter Wohlstand und halbierter Naturverbrauch, München: Droemer Knaur 1995.

(8) Lovins, A.: Natural Capitalism, Cambridge: MIT-Press 1999.

\section{Der Autor}

Dr. Slefan Schaltegger ist Professor für Betriebswirtschafislehre, insbes. Umwelimanagement an der Universiläi lüneburg.

Kontakt: Universität lüneburg, Scharnhorststr. I, 21335 Lüneburg. Tel. 04131/ 782-180, Fax -186, E-mail: schaliegger@uni-lueneburg.de

\section{Literatur zum Schwerpunkt}

Ayres, Robert U./T. van Leynseele: Eco-Efficiency, Double Dividend and the Sustainable Firm. Fontainebleau 1997.

- Behrendt, Siegfried/ Ralf Pfitzner: Ökologische Diensileistungskonzepte als Elemente einer effizienten Kreislaufwirlschaft. Berlin 1998.

- BMBF-Verbundprojekt: Öko-effiziente Dienstleistungen als strategischer Wettbewerbsfaktor zur Entwicklung einer nachhaltigen Wirtschaft. Wuppertal 1998.

- Brüggemonn, Guido: Funktionenorientierung in kulIurellen Perspektiven. Dynamische Wirkungsgefüge von Produktkulturen als neue Basis funktionsorientierter Unternehmenspolitik. Oldenburg 1998.
De Simone, L. D./ F. Popoff: Eco-efficiency. The Business link to Sustainable Development. Cambridge 1997 - Fussler, Claude with Peter James: Driving Eco-Innovation. A Breakthrough Discipline for Innovation and Sustainability. London et al. 1996.

Noorman, K. / J. Uiterkamp/ T. Schoot (Hisg.): Green Households? Domestic Consumers, Environment and Sustainability. London.

OECD: Eco-Efficiency. Paris 1997.

Schmidt-Bleek, Friedrich: Das MIPS-Konzept. Weniger Umweltverbrauch - mehr Lebensqualitär durch Faktor 10. München 1998.

Scholl, Gerd/ Bernd Hirschl / Frank Tibitanzl: Produkie lönger und intensiver nutzen. Grundlogen der
Bewertung never Nutzungskonzepte für Produkte. IÖWSchrittenreihe 134/98, Berlin.

O Schrader, UIf: Empirische Einsichten in die Konsumentenakzepłanz öko-effizienter Diensileistungen. Honnover 1998

Von Weizsïcker, E.U. / A. Lovins/ H. Lovins: Faktor vier: doppelter Wohlstand, halbierter Naturverbrauch. Darmstadt 1995.

WBCSD: Achieving Eco-efficiency in Business. Report of the World Business Council for Sustainable Development - Second Antwerp Eco-efficiency Workshop, March $14-15,1995$

WBCSD/UNEP: Eco-Efficiency and Cleaner Production. Paris. 
(c) 20I0 Authors; licensee IÖW and oekom verlag. This is an article distributed under the terms of the Creative Commons Attribution Non-Commercial No Derivates License (http://creativecommons.org/licenses/by-nc-nd/3.o/), which permits unrestricted use, distribution, and reproduction in any medium, provided the original work is properly cited. 